$$
\alpha_{x} \dot{X}+\alpha_{y} \dot{Y}+\alpha_{z} \dot{Z}-i \dot{X}_{4}=c
$$

which means that the velocity operator on the left of this equation has the proper value equal to the velocity of light. Finally, these expressions suggest that a solution of certain difficulties, which appear to indicate the necessity of a quantized space-time, is to be found, not in an intrinsic property of the space of physics, which possibly has no properties except those given to it by the geometer, but in an intrinsic property of matter.

Bedford College,

University of London. Sept. 28.

1 Snyder, Phys. Rev., 71, 1, 38 (1947).

2 Flint, Phys. Rev., 74, 2, 209 (1948).

${ }^{3}$ Flint, Proc. Roy. Soc., A, 185, 22 (1945).

\section{H. T. FLINT}

\section{Pulse Excitation of Impedance Bridges}

IN the measurement of resistance in a Wheatstone bridge it is customary to use either D.C. or sinusoidal A.c. as excitation. The former is inconvenient if it is desired to use a valve amplifier as the bridge detector; the latter requires a balance of the residual quadrature component and does not indicate directly the sign of the out-of-balance condition of the bridge.

It has been found that for certain purposes the use of rectangular pulses of suitable length and recurrence frequency as excitation for a bridge offers some advantages. In the case of a resistive bridge with stray capacitance shunting one or more arms, the presence of the capacitance causes a spike with an exponential decay at the beginning and end of the pulse, which appears at the output terminals of the bridge, while the plateau which follows the spike gives a correct indication of the resistive balance condition. Investigation shows that measurements can be made in many practical cases with pulses of from 1 to 10 microsec.; while with sinusoidal excitation under similar conditions frequencies as low as $150 \mathrm{c} . / \mathrm{s}$. may be required to make the quadrature component in the bridge output negligible.

One of the most important advantages of the method is that it enables a number of bridges to be excited in sequence and their output applied to a common amplifier. This is the subject of a provisional patent application (J. G. Yates and Elliott Bros. (London), Ltd., No. 22756/47) in connexion with multi-channel resistance strain-gauge equipment. In particular, this avoids the problems of low-level electronic switching for the bridge outputs and re. places it by the easier problem of pulsing the bridge inputs.

The use of pulse excitation is not restricted to the measurement of resistance. For example, the use of pulse excitation in a capacity bridge can provide a convenient method of distinguishing between series and shunt losses in the condenser without requiring measurements at several frequencies.

Some experimental work has been done in collaboration with the Research Laboratories of Elliott Bros. (London), Ltd., and it is hoped to publish further details later.

Engineering Laboratory,

J. G. YATES

University, Cambridge. July 23.

\section{Acclimatization to Cold in the Antarctic}

DURING a year spent as medical officer to the Falkland Islands Dependencies Survey in the Antaretic at Marguerite Bay, I had the opportunity of making some observations on cold acclimatization in eleven men.

In winter and spring, the temperature varied mainly between $0^{\circ}$ and $-35^{\circ} \mathrm{F}$., though in summer temperatures just above freezing were common in the day-time. It was found that as the weather grew progressively colder with the advent of winter, little additional clothing was required. A fair amount of individual variation in clothing worn was noted, though for any given individual little increase in clothing was necessary. One man, for example, wore a string vest, long woollen underpants, an army shirt, silk scarf, socks, boots, gloves, cap with protection for the ears, thin windproof trousers and anorak (windproof upper garment with hood). This was worn for outdoor walking at temperatures down to $0^{\circ} \mathrm{F}$. In colder weather later in the year, a longsleeved sweater, warmer footgear and gloves were all that was necessary in addition. Apart from the footwear and protection for the ears, this was less than the same man was accustomed to wear in England in winter.

In winter, very exceptionally, the temperature rose from the region of $-20^{\circ} \mathrm{F}$. to $+32^{\circ} \mathrm{F}$. overnight, and most of the men complained of the dis. comfort of this relative heat wave.

Difficulties in attempting physiological research on a polar expedition are legion and account for a curtailment of the programme of investigation.

Some of the investigations were to verify interesting results reported by Frazier of the United States Antarctic Service ${ }^{1}$, who quotes Lockhart's work. It was found, for example, that the basal metabolic rate in winter at the base in the Ross Sea was lowered paradoxically. Siple has attributed this low rate to a hibernation mechanism. It is, however, logical that in low environmental temperatures the basal metabolic rate should be raised to provide a greater output of body heat. It is a well-established fact that there is a marked rise in the metabolic rate when an animal is chilled ${ }^{2,3}$, and this rise is attributable to an increased secretion of adrenaline with increased muscular tone and shivering ${ }^{3,4}$. Many observers have recorded a seasonal change, the basal metabolic rate being raised in winter and lowered in summer, Martin ${ }^{6}$ has observed a lowered basal metabolic rate in the tropics. Crill ${ }^{7}$ records a raised basal metabolic rate in Eskimo, but this may be due to the specific dynamic action of a meat diet. Basal metabolic rate estimations made in winter in the Antarctic at Marguerite Bay showed an average increase of 5 per cent over a set of normal contrals with the same apparatus. All estimations were made with the subject comfortably warm in bed. A likely explanation of the discrepancy between these results and those of Lockhart may be that the difference is due to a relative overheating of the United States Antarctic Service huts.

Correlated with a raised basal metabolic rate and increased adrenaline activity, one might expect a raised blood-sugar level, as is found in animal experiments $^{8}$. Lockhart is reported to have found a raised blood-sugar level in his subjects ${ }^{1}$. I found an average increase of $10 \mathrm{mgm}$. per cent in the fasting bloodsugar, determined by the method of Hagedorn and Jensen, in the comfortably warm subject in winter in the Antarctic. 